$\underline{\underline{\beta}}=$ 离

\title{
A single-centered study of factors affecting colistin related nephropathy
}

\author{
Güzin Çakmak $^{1 *}$, Zeynel Abidin Öztürk ${ }^{1}$ \\ ${ }^{1}$ First and Corresponding Author: Guzin Cakmak, MD Gaziantep University, Faculty of Medicine, Department of Internal Medicine, \\ Division of Geriatric Medicine, 27100 Sahinbey, Gaziantep, Turkey \\ *Corresponding author E-mail: drguzincakmak@gmail.com
}

\begin{abstract}
Background: Colistin is an old antibiotic and used for resistant gram-negative infections. Nephrotoxicity is the most common side effect and restricts clinicians to use it. Many risk factors suggested for the development of nephropathy. It could be better to know risks to prevent nephropathy development. Objectives: In this study, we aimed to evaluate factors that affecting the development of nephropathy in patients who were hospitalized in hematology, oncology, palliative care, and intensive care units. Methods: 125 patients aged 20-93 years old were enrolled in this retrospective study. Data were obtained from the pharmacy of the university hospital and information management system of the hospital. Results: Among 125 patients included in the study, 15 developed KDIGO stage 1 nephropathy, 21 stages two nephropathies, and four stages three nephropathies, respectively. Age, albumin level, and bilirubin levels were shown to be related to nephropathy. We found the cut off value of albumin $3.05 \mathrm{mg} / \mathrm{dl}$ for development of nephropathy (sensitivity $70 \%$; specificity $66 \%$; $\mathrm{CI}=95 \%, \mathrm{p}=0.003$ ) and mortality (sensitivity 69\%; specificity 61\%; $\mathrm{CI}=95 \%, \mathrm{p}<0.001$ ). We revealed that age and bilirubin levels independently affected the development of nephropathy in linear regression analysis $(\mathrm{r} 2=0.131, \mathrm{p}=0.032 ; \mathrm{p}=0.015)$. Also, mortality was denoted to be related to albumin and bilirubin levels independently in linear regression analysis $(r 2=0.161, p=0.03$; $p=0.004)$. Conclusions: Older age, hypoalbuminemia, and hyperbilirubinemia were risk factors for colistin related nephropathy and mortality. Further studies will be better to elucidate relationships more clearly.
\end{abstract}

Keywords: Colistin; Nephropathy; Colistin Nephropathy; Colistin Related Nephropathy.

\section{Introduction}

Polymyxins are cationic cyclic polypeptide antibiotics that identified as fermentation products of the bacteria Bacillus polymyxa(Yu et al. 2018). Their antimicrobial activity was first recognized in the 1940s (Lim et al. 2015). There are five groups of polymyxins, starting from polymyxin A to polymyxin E (\&NA; 2005). Polymyxin E or colistin is useful for the treatment of infections caused by resistant microorganisms like Pseudomonas aeruginosa, Acinetobacter baumannii, Klebsiella pneumonia, and Escherichia coli (Çetin et al. 2016). Bacterial resistance against the drug is not as broad as others like cephalosporins, quinolones, and carbapenems (Das, Goel, and Bhattacharya 2018). In the 1970s, nephrotoxicity and neurotoxicity of colistin attracted attention. Its use was stopped. Instead of colistin, newer gram-negative antibacterial agents that were perceived to be less toxic were preferred to use for resistant gram-negative infections. However, the emergence of multi-drug resistant microorganisms (MDRO) made use of colistin necessary again (Hachem et al. 2007). Gram-negative bacteria have a three-layered envelope, including the outer membrane (OM), the cytoplasmic membrane, and a peptidoglycan layer in the periplasmic space. The outer membrane of gram-negative bacteria has an amphipathic structure (Clarke, Gray, and Reaveley, 1967). Divalent cations like $\mathrm{Mg}^{2+}$ and $\mathrm{Ca}^{2+}$ are responsible for maintaining lipopolysaccharide (LPS) structure (Clifton et al. 2015). Polymyxins have a high affinity for LPS molecules because of their cationic structure (Morrison and Jacobs 1976). One of the mechanisms that explain the polymyxins bactericidal effect on gram-negative bacteria is based on this interaction. Polymyxin binding causes the displacement of divalent cations that function as stabilizers of OM of gram-negative bacteria. Disturbance of LPS structure allows more polymyxins to transverse OM (Acta, Schindler, and Osborn 1979). Some other mechanisms that explain bactericidal activity are the formation of molecular connections between OM and cytoplasmic membrane, perforation of the cell wall, loss of intracellular proteins, and production of reactive oxygen species (O'Driscoll et al. 2018).

The major drawback of colistin use for resistant gram-negative infections is nephrotoxicity. To prevent the development of colistin induced nephrotoxicity, defining risk factors is essential. Some of them are increased age, comorbid conditions, prolonged time of treatment, high doses, use of other nephrotoxic agents, pre-existing renal failure, hypertension, and obesity (Shields et al. 2017). In addition to these factors, hyperbilirubinemia and hypoalbuminemia are some other risk factors suggested to be related to colistin nephrotoxicity (E. J. Kim and Kim 2018).

In this study, we evaluated the relationship between hyperbilirubinemia, hypoalbuminemia, colistin nephrotoxicity, and mortality. 


\section{Methods}

\subsection{Study design and collection of data}

This single-centered, retrospective study was conducted at the Gaziantep University Faculty of Medicine. One hundred twenty-five patients were enrolled in the study. Their age was between 20-93 years old; the mean age was $51.5 \pm 1.9$. In this study, patients who were hospitalized in units of palliative care, medical oncology, hematology, and intensive care unit between years of 2018-2019 evaluated. Exclusion criteria were; missing data, colistin use less than 48 hours, and hemodialysis. Only patients that took colistin intravenously were enrolled to study. The development of acute kidney injury and mortality were end-points of the study. Patients' ID and numbers were taken from the pharmacy of the university hospital. We obtained patients' clinical and demographical information from the information management system of the hospital.

\subsection{Colistin treatment}

Intravenous colistimethate sodium (CMS) was used in this study. The administration of CMS for multi-drug resistant infection was based upon the results of in vitro antimicrobial susceptibility tests. $2.5-5.0 \mathrm{mg} / \mathrm{kg} / \mathrm{day}$ (not to exceed $5 \mathrm{mg} / \mathrm{kg} / \mathrm{day}$ ) CMS was used for patients with normal renal function. The total daily dosage was reduced when renal impairment was seen, according to the manufacturer's instructions. Treatment duration was determined due to clinical response.

\subsection{Definition of development of renal injury}

Acute kidney injury was defined by using creatinine levels according to the Kidney Disease: Improving Global Outcomes (KDIGO) criteria (Machado, Nakazone, and Maia 2014).

\subsection{Blood collection and biochemical analysis}

Blood was collected from patients after 8 hours of night fasting in the morning. Venous blood was taken from the antecubital vein. Creatinine, albumin, and bilirubin levels were measured in serum by commercial test kits in a biochemistry auto-analyzer (Beckman Coulter).

\subsection{Statistics}

The IBM SPSS Statistics 22 software program was used to analyze data. Continuous variables of groups were compared by using the independent sample t-test. Relationships between parameters were investigated by Pearson correlation analysis. Also, the linear regression model was used to analyze relationships between parameters. ROC (Receiver Operating Characteristic) analysis was done for determining cut off values of parameters that predicted nephropathy and mortality. The statistical significance level was determined as $\mathrm{p}<0.05$.

\subsection{Ethical approval and informed consent}

No specific, informed consent or ethical approval needed for the retrospective study

\section{Results}

There were 125 patients, and these patients were investigated about colistin nephropathy and factors influencing colistin nephropathy. Eighty-five of them were hospitalized in the hematology clinic, 15 of them in the medical oncology clinic, 16 of them in the palliative care unit, 9 of them in the intensive care unit. Primary diagnoses of the patients were summarized in table-1. 59\% of them ( $\mathrm{n}=74)$ were male and $41 \%$ of them $(n=51)$ were female. Thirty of them were $\geq 65$ years old. Thirty-eight of them were infected with Klebsiella pneumonia, 35 of them with Acinetobacter baumanii, 29 of them with Escherichia coli, and 23 of them with Pseudomonas aeruginosa. Eightyfive of the patients did not develop nephropathy; 15 of them developed stage 1 nephropathy, 21 of them developed stage 2 nephropathy, and 4 of them developed stage 3 nephropathy. Age was demonstrated to be related to nephropathy and mortality. Age was also negatively related to albumin level $(\mathrm{p}=0.002, \mathrm{r}=-0.266)$. Our study revealed that serum albumin levels were inversely related to nephropathy and mortality. Also, we revealed that the bilirubin level was related to nephropathy and mortality. Results were summarized in table- 2 . We determined the cut off value of albumin $3.05 \mathrm{mg} / \mathrm{dl}$ for development of nephropathy (sensitivity 70\%; specificity $66 \%$; CI=95\%, $\mathrm{p}=0.003$ ) (figure-1) and mortality (sensitivity 69\%; specificity $61 \%$; $\mathrm{CI}=95 \%, \mathrm{p}=0.00$ ) (figure-2). Nephropathy was strongly related to mortality $(\mathrm{p}<0.001, \mathrm{r}=0.400)$. Gender was not related to nephropathy $(\mathrm{p}=0.051)$ or mortality $(\mathrm{p}=0.66)$. Also, there was no relationship between age, albumin, bilirubin, and gender (table-3). In linear regression analysis, age and bilirubin levels were found to be independently related with nephropathy $\left(\mathrm{r}^{2}=0.131, \mathrm{p}=0.032 ; \mathrm{p}=0.015\right)$. Albumin level was found to be independently associated with nephropathy when we did linear regression analysis with albumin level and bilirubin level $\left(\mathrm{r}^{2}=0.096, \mathrm{p}=0.028\right)$, but when we added age to parameters albumin level became not related with nephropathy $\left(r^{2}=0.131, p=0.124\right)$. Mortality was found to be related to albumin and bilirubin level in linear regression analysis too $\left(\mathrm{r}^{2}=0.161, \mathrm{p}=0.03 ; \mathrm{p}=0.004\right)$. Age was found not to be related to mortality in linear regression analysis $\left(r^{2}=0.161, p=0.087\right)$. We did not evaluate the relationship between colistin nephrotoxicity and using other nephrotoxic drugs because other than seven patients, all of them were using concomitant nephrotoxic drugs. The number of nephrotoxic drugs used by patients was shown in table-4.

\section{Discussion}

In this study, we revealed that the presence of hypoalbuminemia, hyperbilirubinemia, and aging increased the probability of colistin nephropathy development. These factors also increased mortality. Previous studies also showed us that low albumin levels were related to nephropathy. Levels below $2 \mathrm{mg} / \mathrm{dl}$ (milligram/deciliter), $2.5 \mathrm{mg} / \mathrm{dl}$, and $3.2 \mathrm{mg} / \mathrm{dl}$ were found to be related to nephrotoxicity in differ- 
ent studies (Kwon et al. 2010)(Giacobbe et al. 2018)(J. Kim et al. 2009). Many mechanisms being used to explain the relationship between hypoalbuminemia and colistin nephrotoxicity. One of them is dependent on albumin-bound and unbound fractions of colistin. An unbound fraction of colistin is suggested to be responsible for the development of nephrotoxicity (Mohamed et al. 2012). Another mechanism is dependent on antioxidant status. Antioxidant properties of serum albumin and its free-radical trapping activity were mentioned in previous studies (Taverna et al. 2013). Some authors suggest that reduced antioxidant capacity due to hypoalbuminemia causes increased proximal tubular damage(De Castro et al. 2014). Also, diminished renal perfusion as a result of hypoalbuminemia is thought to be a facilitator of colistin nephropathy(Wiedermann, Wiedermann, and Joannidis 2017). Finally, albumin is a well-known negative acute phase reactant(Ahmed et al. 2012). Hypoalbuminemia can indicate both inflammation and malnutrition, and it can be associated with poor health status (Jellinge et al. 2014). Multiple factors that contribute to poor health status can also influence renal function.

Nevertheless, in our study, we also encountered a different result; in linear regression analysis, we found that albumin and bilirubin affected the development of nephropathy independently from each other, but when age was included to analyze albumin became not an independent factor. Also, we found a negative relationship between age and albumin levels. So the relationship between albumin level and nephropathy seems to be affected by age. On the other hand, mortality was found to be related to albumin and bilirubin, and unexpectedly age was found not to be related to mortality in linear regression analysis. It might be related to that number of elderly patients was only 30 .

In our study, we also revealed the effect of hyperbilirubinemia on colistin renal toxicity. The relationship between jaundice and renal failure has been known for a long time. Jaundice can cause tubular injury-induced electrolyte disturbances (e.g., hyponatremia, hypokalemia, hypouricemia, normal gap metabolic acidosis, and Fanconi syndrome) and acute kidney injury. Bile acids are directly toxic to tubular cells (Wardle 1975). Also, bile salts can cause nephropathy by accumulating in the tubular lumen. This situation is called as bile cast nephropathy or cholemic nephrosis. The mechanism is similar to the mechanism of cast nephropathy in multiple myeloma (Jain et al. 2015). In Kwon et al.'s retrospective study, male sex was revealed as an independent risk factor of colistin nephropathy, although in our study relationship between gender and nephropathy was not found. In the same study, hypoalbuminemia and hyperbilirubinemia were defined as independent risk factors for colistin related nephropathy as in our study (Kwon et al. 2010).

Many mechanisms were supposed to explain colistin nephrotoxicity. One of them is the detergent theory. They supposed that colistin sulfate interacted with anionic phospholipids and disturbed cell membrane permeability(Gai et al. 2019).

Another theory is that the cause of colistin nephrotoxicity is the accumulation of colistin in proximal tubular cells of the kidney (Suzuki et al. 2013).

Our study is critical because few studies were investigating the effect of age, albumin, and bilirubin on colistin nephropathy together. It could be better to evaluate the relationship between the duration of hospitalization and other parameters. Also, we denoted that the albumin effect on nephropathy might be influenced by age. Only 30 of our patients were elderly patients, and it could be better to evaluate the effect of age in a population that was more balanced upon age distribution. Besides these, we found a new cut-off value of albumin for colistin nephropathy development and mortality.

Colistin is an antimicrobial that has a narrow therapeutic range, commonly used for multi-drug resistant infections, and that infections are seen in patients who have a critical illness. Hypoalbuminemia and hyperbilirubinemia can be seen because of many factors in those patients. So the effect of hypoalbuminemia and hyperbilirubinemia on colistin nephropathy can be affected by multiple factors. Evaluation and differentiation of those factors may be hard. The major limitation of our study was that situation. Another limitation of our study was that we could not be able to evaluate the effect of albumin replacement on albumin levels, development of nephropathy, and mortality retrospectively.

\section{Conclusion}

In this study, we revealed that older age, hypoalbuminemia, and hyperbilirubinemia were risk factors for colistin related nephropathy and mortality. Correcting albumin levels or avoidance of colistin use in hyperbilirubinemia patients can be useful for diminishing rate of colistin related nephropathy. Also, we reached the result that colistin should be used more carefully in older patients.

\section{Conflict of interest statement}

Zeynel Abidin Öztürk declared that he has no conflict of interest

\section{Acknowledgments}

I would like to express my special thanks of gratitude to Professor Zeynel Abidin Ozturk and all my colleagues that we worked together in this study.

Abbreviations

CI: cumulative index

OM: outer membrane

LPS: lipopolysaccharide

KDIGO: Kidney Disease: Improving Global Outcomes

ROC: Receiver Operating Characteristic

NSAID: non-steroidal anti-inflammatory drug

Table 1: Primary Diagnosis of Patients

\begin{tabular}{llll} 
& Table 1: Primary Diagnosis of Patients & Percent $(\%)$ \\
\hline & & frequency & 36.8 \\
& Leukemia & 46 & 18.4 \\
Diagnosis & Multiple myeloma & 23 & 16.8 \\
& Solid-organ tumor & 21 & 13.6 \\
& Lymphoma & 17 & 11.2 \\
& Alzheimer's disease & 14 & 3.2 \\
& Cerebrovascular event & 4 & 100 \\
\hline
\end{tabular}


Table 2: Factors Affecting Mortality and Nephropathy

\begin{tabular}{lll}
\hline & Nephropathy & Mortality \\
\hline Age & $\mathrm{p}=0.00, \mathrm{r}=0.326$ & $\mathrm{p}=0.01, \mathrm{r}=0.233$ \\
Albumin $(\mathrm{g} / \mathrm{dL})$ & $\mathrm{p}=0.003, \mathrm{r}=-0.266$ & $\mathrm{p}=0.004, \mathrm{r}=-0.258$ \\
Bilirubin $(\mathrm{mg} / \mathrm{dL})$ & $\mathrm{p}=0.005, \mathrm{r}=0.251$ & $\mathrm{p}=0.002, \mathrm{r}=0.277$ \\
\hline
\end{tabular}

Table 3: Relationship between Parameters and Gender

\begin{tabular}{llll}
\hline Gender & female $($ mean \pm SD) & male $($ mean \pm SD) & $\mathrm{p}=$ \\
\hline Age & $54 \pm 20$ & $50 \pm 18$ & 0.241 \\
Albumin & $3.12 \pm 0.6$ & $2.97 \pm 0.8$ & 0.244 \\
Bilirubin & $1.74 \pm 0.56$ & $1.17 \pm 0.23$ \\
\hline
\end{tabular}

Table 4: Nephrotoxic Drugs Used with Colistin

\begin{tabular}{ll}
\hline Drug name & Number of patients used \\
\hline Meropenem & 77 \\
Valacyclovir & 63 \\
Daptomycin & 47 \\
Amikacin & 21 \\
Cyclosporin & 17 \\
Cefalosporin & 6 \\
NSAID & 4 \\
Diuretic & 4 \\
Others & 7 \\
\hline
\end{tabular}

NSAID: the non-steroidal anti-inflammatory drug.

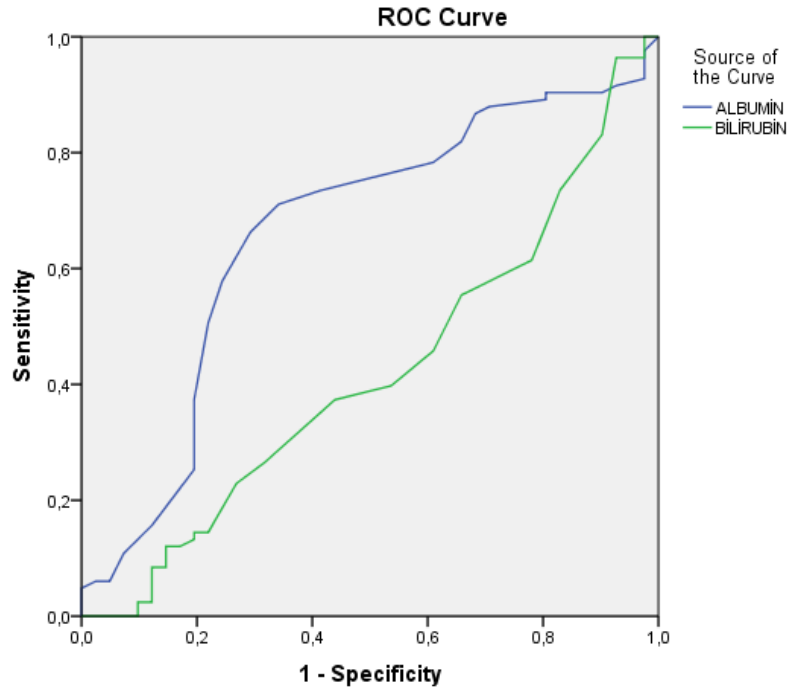

Diagonal segments are produced by ties

Fig. 1: ROC Curve of Nephropathy.

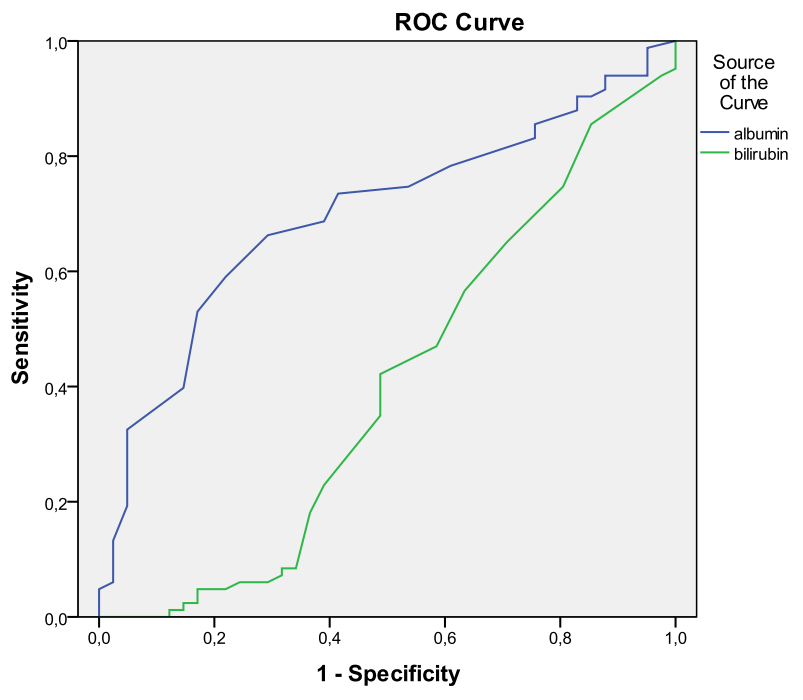

Diagonal segments are produced by ties.

Fig. 2: ROC Curve of Mortality. 


\section{References}

[1] \&NA; 2005. "Colistin: The Revival of Polymyxins for the Management of Multidrug-Resistant Gram-Negative Bacterial Infections." The Pediatric Infectious Disease Journal 24(10): 945. https://doi.org/10.1097/01.inf.0000174577.97635.7b.

[2] Acta, J Biochim Biophys, Melvin Schindler, and M J Osborn. 1979. "Interaction of Divalent Cations and Polymyxin B With.” 18(20): 4425-30. https://doi.org/10.1021/bi00587a024.

[3] Ahmed, M. S., A. B. Jadhav, A. Hassan, and Qing H. Meng. 2012. "Acute Phase Reactants as Novel Predictors of Cardiovascular Disease." ISRN Inflammation 2012: 1-18. https://doi.org/10.5402/2012/953461.

[4] De Castro, Lais Lima et al. 2014. "Hipoalbuminemia y El Estres Oxidativo En Pacientes En Programa de Dialisis Renal." Nutricion Hospitalaria 30(4): 952-59.

[5] Çetin, Çiğdem Banu et al. 2016. "Colistin Efficacy in the Treatment of Multidrug-Resistant and Extremely Drug-Resistant Gram-Negative Bacterial Infections.” Turkish Journal of Medical Sciences 46(5): 1379-84. https://doi.org/10.3906/sag-1506-125.

[6] Clarke, K., G. W. Gray, and D. A. Reaveley. 1967. "The Cell Walls of Pseudomonas Aeruginosa. General Composition.” The Biochemical journal 105(2): 749-54. https://doi.org/10.1042/bj1050749.

[7] Clifton, Luke A. et al. 2015. "Effect of Divalent Cation Removal on the Structure of Gram-Negative Bacterial Outer Membrane Models." Langmuir 31(1): 404-12. https://doi.org/10.1021/la504407v.

[8] Das, Parijat, Gaurav Goel, and Sanjay Bhattacharya. 2018. "Official Publication of Academy of Clinical Microbiologists.” (January).

[9] Gai, Zhibo, Sophia L. Samodelov, Gerd A. Kullak-Ublick, and Michele Visentin. 2019. "Molecular Mechanisms of Colistin-Induced Nephrotoxicity." Molecules (Basel, Switzerland) 24(3). https://doi.org/10.3390/molecules24030653.

[10] Giacobbe, Daniele Roberto et al. 2018. "Hypoalbuminemia as a Predictor of Acute Kidney Injury during Colistin Treatment." Scientific Reports 8(1): 1-11. https://doi.org/10.1038/s41598-018-30361-5.

[11] Hachem, Ray Y. et al. 2007. "Colistin Is Effective in Treatment of Infections Caused by Multidrug-Resistant Pseudomonas Aeruginosa in Cancer Patients." Antimicrobial Agents and Chemotherapy 51(6): 1905-11. https://doi.org/10.1128/AAC.01015-06.

[12] Jain, Koyal et al. 2015. "Bile Cast Nephropathy." Kidney International 87(2): 484. https://doi.org/10.1038/ki.2014.233.

[13] Jellinge, Marlene Ersgaard, Daniel Pilsgaard Henriksen, Peter Hallas, and Mikkel Brabrand. 2014. "Hypoalbuminemia Is a Strong Predictor of 30Day All-Cause Mortality in Acutely Admitted Medical Patients: A Prospective, Observational, Cohort Study." PLoS ONE 9(8): 8-12. https://doi.org/10.1371/journal.pone.0105983.

[14] Kim, Eun Jung, and Eu Suk Kim. 2018. "Exploring New Predictors of Colistin-Associated Nephrotoxicity." Infection and Chemotherapy 50(3): 283-85. https://doi.org/10.3947/ic.2018.50.3.283.

[15] Kim, Jieun, Kyoung Ho Lee, Sunmi Yoo, and Hyunjoo Pai. 2009. "Clinical Characteristics and Risk Factors of Colistin-Induced Nephrotoxicity." International Journal of Antimicrobial Agents 34(5): 434-38. https://doi.org/10.1016/j.ijantimicag.2009.06.028.

[16] Kwon, Jeong Ah et al. 2010. "Predictors of Acute Kidney Injury Associated with Intravenous Colistin Treatment." International Journal of Antimicrobial Agents 35(5): 473-77. https://doi.org/10.1016/j.ijantimicag.2009.12.002.

[17] Lim, Lauren M et al. 2015. "HHS Public Access.” 30(12): 1279-91.

[18] Machado, Maurício Nassau, Marcelo Arruda Nakazone, and Lilia Nigro Maia. 2014. "Lesão Renal Aguda Baseada Nos Critérios KDIGO (Kidney Disease: Improving Global Outcomes) Em Pacientes Com Creatinina Sérica Elevada Submetidos à Cirurgia Cardíaca." Brazilian Journal of Cardiovascular Surgery 29(3): 299-307.

[19] Mohamed, Ami F. et al. 2012. "Application of a Loading Dose of Colistin Methanesulfonate in Critically Ill Patients: Population Pharmacokinetics, Protein Binding, and Prediction of Bacterial Kill.” Antimicrobial Agents and Chemotherapy 56(8): 4241-49. https://doi.org/10.1128/AAC.0642611.

[20] Morrison, David C., and Diane M. Jacobs. 1976. "Binding of Polymyxin B to the Lipid A Portion of Bacterial Lipopolysaccharides." Immunochemistry 13(10): 813-18. https://doi.org/10.1016/0019-2791(76)90181-6.

[21] O’Driscoll, Noëlle H., T. P.Tim Cushnie, Kerr H. Matthews, and Andrew J. Lamb. 2018. "Colistin Causes Profound Morphological Alteration but Minimal Cytoplasmic Membrane Perforation in Populations of Escherichia Coli and Pseudomonas Aeruginosa." Archives of Microbiology 200(5): 793-802. https://doi.org/10.1007/s00203-018-1485-3.

[22] Shields, Ryan K. et al. 2017. "Defining the Incidence and Risk Factors of Colistin-Induced Acute Kidney Injury by KDIGO Criteria." PLoS ONE 12(3): 3-7. https://doi.org/10.1371/journal.pone.0173286.

[23] Suzuki, Takahiro et al. 2013. "Megalin Contributes to Kidney Accumulation and Nephrotoxicity of Colistin." Antimicrobial Agents and Chemotherapy 57(12): 6319-24. https://doi.org/10.1128/AAC.00254-13.

[24] Taverna, Myriam, Anne Lise Marie, Jean Paul Mira, and Bertrand Guidet. 2013. "Specific Antioxidant Properties of Human Serum Albumin." Annals of Intensive Care 3(1): 1-7. Annals of Intensive Care. https://doi.org/10.1186/2110-5820-3-4.

[25] Wardle, E. N. 1975. "Renal Failure in Obstructive Jaundice-Pathogenic Factors." Postgraduate Medical Journal 51(598): 512-14. https://doi.org/10.1136/pgmj.51.598.512.

[26] Wiedermann, Christian J, Wolfgang Wiedermann, and Michael Joannidis. 2017. "Causal Relationship between Hypoalbuminemia and Acute Kidney Injury." World Journal of Nephrology 6(4): 176. https://doi.org/10.5527/wjn.v6.i4.176.

[27] Yu, Zhiliang, Zhongqi Sun, Jianhua Yin, and Juanping Qiu. 2018. "Enhanced Production of Polymyxin E in Paenibacillus Polymyxa by Replacement of Glucose by Starch.” BioMed Research International 2018. https://doi.org/10.1155/2018/1934309. 